\title{
BM] Global Health Predictors of attrition from care at 2 years in a prospective cohort of HIV- infected adults in Tigray, Ethiopia
}

Raffaella Bucciardini, ${ }^{1}$ Vincenzo Fragola, ${ }^{1}$ Teshome Abegaz, ${ }^{2}$ Stefano Lucattini, ${ }^{1}$ Atakilt Halifom, ${ }^{3}$ Eskedar Tadesse, ${ }^{2}$ Micheal Berhe, ${ }^{2}$ Katherina Pugliese, ${ }^{1}$ Luca Fucili, ${ }^{1}$ Massimiliano Di Gregorio, ${ }^{1}$ Marco Mirra, ${ }^{1}$ Paola De Castro, ${ }^{1}$ Roberta Terlizzi, ${ }^{1}$ Paola Tatarelli, ${ }^{4}$ Andrea Binelli, ${ }^{1}$ Teame Zegeye, ${ }^{3}$ Michela Campagnoli, ${ }^{1}$ Stefano Vella, ${ }^{1}$ Loko Abraham, ${ }^{2}$ Hagos Godefay ${ }^{3}$

To cite: Bucciardini R, Fragola V, Abegaz T, et al. Predictors of attrition from care at 2 years in a prospective cohort of HIV-infected adults in Tigray, Ethiopia. BMJ Glob Health 2017;2:e000325. doi:10.1136/ bmjgh-2017-000325

Received 20 February 2017 Revised 17 May 2017 Accepted 29 June 2017
CrossMark

${ }^{1}$ Istituto Superiore di Sanità, Rome, Italy

${ }^{2}$ College of Health Sciences, Mekelle University, Mekelle, Ethiopia

${ }^{3}$ Tigray Regional Health Bureau, Mekelle, Ethiopia

${ }^{4}$ Department of Infectious Diseases, Università degli studi di Genova, Genoa, Italy

Correspondence to Dr Raffaella Bucciardini; raffaella.bucciardini@iss.it

\section{ABSTRACT}

Introduction Ethiopia has experienced rapid expansion of antiretroviral therapy (ART). However, as long-term retention in ART therapy is key for ART effectiveness, determinants of attrition need to be identified so appropriate interventions can be designed.

Methods We used data from the 'Cohort of African people Starting Antiretroviral therapy' (CASA) project, a prospective study of a cohort of HIV-infected patients who started ART in seven health facilities (HFs). We analysed the data of patients who had started first-line ART between January 2013 and December 2014. The Kaplan-Meier method was used to estimate the probability of retention at different time points. The Cox proportional hazards model was used to identify factors associated with attrition.

Results A total of 1198 patients were included in the study. Kaplan-Meier estimates of retention in care were $83.9 \%, 82.1 \%$ and $79.8 \%$ at 12,18 and 24 months after starting ART, respectively. Attrition was mainly due to loss to follow-up, transferred-out patients and documented mortality. A multivariate Cox proportional hazard model showed that male sex, CD4 count $<200$ cells/ $\mu \mathrm{L}$ and the type of HF were significantly associated with attrition. Conclusions The observed attrition differences according to gender suggest that separate interventions designed for women and men should be explored. Moreover, innovative strategies to increase HIV testing should be supported to avoid CD4 levels falling too low, a factor significantly associated with higher attrition in our study. Finally, specific studies to analyse the reasons for different levels of attrition among HFs are required.

\section{INTRODUCTION}

The expansion of access to antiretroviral therapy (ART) over the last 10 years is a remarkable global health achievement, with over 17 million people living with HIV (PLWH) on ART in 2015, of whom nearly 10 million live in resource-limited settings. ${ }^{1}$ According to the latest Joint United Nations Programme on HIV/AIDS (UNAIDS) data, the effects of scaling up ART were greatest

\section{Summary box}

What is already known about this subject?

- Long-term retention is key for ART effectiveness, both clinically and in terms of reducing HIV transmission.

- Understanding the factors influencing long-term retention rates is crucial for designing effective ART programmes.

- There are are numerous biomedical, social and behavioural determinants of attrition, but they have been often explored using retrospective data analysis.

What are the new findings?

- In a prospective study, we confirmed gender-related differences in attrition.

- Low CD4 levels ( $<200$ cells/ $/ \mu L)$ at enrolment were associated with an increased risk of attrition.

- The level of attrition varies among health facilities.

- Although decentralisation has positive effects on ART delivery, we observed that a high number of patients transferred out to other health facilities without official documentation attesting that they were still on ART.

What are the recommendations for policy and practice?

- The observed gender difference suggests that separate ART programmes should be designed for women and for men.

- The impact of low CD4 levels at enrolment on attrition suggests strategies should be strengthened to increase HIV counselling and testing, followed by quick ART initiation as per WHO guidelines.

- Local policy makers should seek to understand the causes of the observed differences in attrition among health facilities

in the world's most affected regions, eastern and southern Africa. Coverage increased from $24 \%$ ( $22 \%$ to $26 \%$ ) in 2010 to $54 \%$ $(50 \%-58 \%)$ in 2015 and the number of 
AIDS-related deaths has been reduced by $36 \%$ since 2010. In addition, adult HIV infections have decreased by $4 \%$, with about 40,000 fewer new cases of adult HIV infection in 2015 than in $2010 .^{2}$

Ethiopia, with an estimated $730,000(600,000$ to 970,000$)$ PLWH and a HIV prevalence among adults of $1.2 \%$ $(1.0 \%$ to $1.5 \%)$ at the end of 2014 , is one of the sub-Saharan countries which has implemented effective strategies to scale up ART. Since 2005, the Ethiopian government has introduced a programme making ART available on a free basis, with over 362,000 PLWH receiving ART in 2014. As a consequence, since the Millennium Declaration, the country has significantly reduced the incidence of HIV/AIDS as well as the number of deaths due to HIV/ AIDS from $73,000(60,000$ to 93,000$)$ in 2000 to 23,000 $(17,000$ to 41,000$)$ in 2014 . However, despite the progress in scaling up ART, only $50 \%$ (41\%66\%) of HIV-infected people in Ethiopia currently receive ART. ${ }^{3}$

A further expansion of HIV treatment programmes is expected following the 2015 expansion in the WHO ART eligibility criteria, from a CD4 cell count of $<500$ to all HIV patients irrespective of CD4, and introduction of the UNAIDS 90-90-90 strategy. ${ }^{4}$

However, to achieve the 90-90-90 treatment target, there is an urgent need to improve each step of HIV care in order to maximise the individual and public health benefits of ART. Suboptimal adherence and ART retention are a substantial barrier to programme effectiveness. According to recent reports, retention of the adult PLWH population may fall below $50 \%$ at 5 years after ART initiation in sub-Saharan Africa. ${ }^{5}$

Despite universal access to HIV care and treatment, long-term ART retention is a major health challenge in Ethiopia. Maintaining a high level of patient retention in care is key to obtaining personal and public health benefits. Determinants of attrition therefore need to be identified so appropriate interventions can be designed.

This prospective study aims to estimate retention in care and to identify predictors of attrition among patients initiating ART in seven public health facilities (HFs) located in Tigray in northern Ethiopia.

This paper is an extension of a previously published article describing the results of a 1-year follow-up analysis. Compared with the earlier report, this article provides more representative results since a longer follow-up period and a larger sample size have been used. ${ }^{6}$

\section{METHODS}

\section{Study design and setting}

We used data from the 'Cohort of African people Starting Antiretroviral therapy' (CASA) project, a prospective, ongoing, multi-site study of a cohort of HIV-infected patients who started ART in seven urban and rural HFs located in Tigray, the northeastern region of Ethiopia. The HFs participating in the study include two health centres (Alamata Health Centre, Mekelle
Health Centre), one university hospital (Ayder Referral Hospital), three general hospitals (Alamata General Hospital, Mekelle General Hospital and Lemlem Karl General Hospital of Michew) and one primary hospital (Mehoni Primary Hospital). The ART units in the health centres are run by two or three nurses, the primary hospital ART unit has three or four nurses and in some cases one health worker with a master's degree in infectious diseases, and the general hospital ART unit and the referral hospital ART unit have three or four nurses and one medical doctor.

\section{Participants}

HIV-infected adults who fulfilled the WHO criteria for starting ART at the participating HFs were eligible for enrolment in our cohort. All individuals over 14 years of age who had initiated ART between 1 January 2013 and 31 December 2014 and who agreed to provide their home address and a telephone contact number were selected for this study. Follow-up data were available until December 2015.

\section{Ethical considerations}

Ethics approval was obtained from the Health Research Ethics Review Committee of Mekelle University College of Health Science (reference number: ERC 0129/2012). All patients provided written informed consent. For patients aged 14-18, the informed consent form was signed by adult relatives acting as guardians (immediate families, e.g. father or mother or next of kin) and not by the patients themselves.

\section{Outcome measures}

The operational definitions of the outcomes used in this study are as follows:

- Loss to follow-up: patients who missed visits to the same HF for more than 3 months after the last scheduled visit.

- Stop ART medication: patients known to have stopped ART for any reason (personal or clinical decision).

- Mortality: patients recorded as dead on the patient's exit form.

- Transfer out: patients formally transferred to another HF. Transferred-out patients were not considered as retained in care because official documentation to certify that they were still on ART was not available. Transferred patients were considered as retained in the initial HF until the date they were transferred out.

- Retention in care: patients who were alive and receiving ART at the same HF after ART initiation (this does not include patients who were recorded as lost to follow-up (LTFU), who discontinued ART, who were deceased or who transferred out).

- Attrition from care: this is the opposite of retention and included patients who were LTFU, had discontinued ART, were recorded as deceased or had transferred out. 


\section{Independent variables}

The following demographic and clinical variables at enrolment were considered as potential predictors of attrition: gender, age, religion, educational status, WHO clinical stage (from stage I to stage IV), haemoglobin level, body mass index (BMI) calculated as weight $(\mathrm{kg}) /$ height $(\mathrm{m})^{2}$ (underweight $\leq 18.5$, normal 18.6-25, overweight $>25$ ), initial ART regimen, CD4 cell counts (within 6 months of starting HIV treatment) and the presence of active tuberculosis (TB) infection with Mycobacterium tuberculosis where the diagnosis had been made by sputum smear and chest x-ray.

\section{Data source}

The baseline visit and follow-up visits were carried out according to the national guidelines and the standard of care of the participating HFs. Data, including demographic and clinical information as well as the patient's current status (known to be dead, transferred-out, discontinued or LTFU) were systematically collected by ART nurses using forms specifically designed for this study (enrolment form, follow-up form, exit form).

Each HF used desktop software developed in the .Net framework (Microsoft) especially for this study to enter the demographic, clinical and laboratory data of participating patients. Data were first entered by a case manager (CM) from each site and then merged and managed in a central database. It was important for data completeness, reliability and validity to standardise the data collection procedures for all participating sites. Staff members responsible for collecting and entering study data received appropriate training and data monitoring was conducted throughout the study. The project implementation office in Ayder Hospital together with the project coordinating centre (Istituto Superiore di Sanità) in Italy were responsible for oversight of data quality and generating queries (missing data, inconsistencies in the data, potential errors) for each participating HF. The project implementation office staff were responsible for conducting regular site visits to assist local personnel with the queries and ensure procedures were followed appropriately.

\section{Tracing of patients who failed to return for a scheduled visit}

Routine HIV/AIDS care is provided in the participating HFs according to national guidelines. Support for PLWH is regularly provided by local community-based organisations and community health workers (CHWs). In the present study, the active participation of CHWs was enhanced to trace patients as described below. At each participating HF, patient data were entered by a study CM. At the beginning of each week (Monday), the CM prepared a list containing the details of patients who had missed their scheduled visit for more than 1 month. These lists were then given to trained CHWs who traced the patients using telephone interviews, face-to-face contacts or proxy information (family members or friends). Traced patients were asked why they had missed the scheduled visit. CHWs were trained to provide basic information on HIV and ART and on the importance of taking drugs regularly without interruption. Patients were asked to return to the HF and resume ART. At the end of each week (usually on Friday), CHWs returned the list to CMs together with the following information gathered during the week ${ }^{1}$ : whether or not the patient had been traced, ${ }^{2}$ how the patient had been traced (by phone, face to face, through other people),$^{3}$ the current vital status (alive or death) of the traced patient, ${ }^{4}$ patient intent to return to the HF, and the reasons for his/her decision.

\section{Statistical methods}

The primary outcome measure was retention in care 2 years after ART initiation. The Kaplan-Meier method was used to estimate the probability of retention in care, mortality, loss to follow-up, transfer out, and stopping ART medication at different time points. Univariate and multivariate Cox proportional hazards models with robust sandwich estimates, to account for within-HF correlation, were used to identify factors associated with attrition. All available demographic and clinical variables were considered as potential predictive factors and were included in the univariate analysis: type of HF, gender, age (14-25, 26-50, >50 years), educational status (no education, primary, secondary, tertiary), religion (Orthodox Christian or other religion), BMI $(\leq 18.5,18.6-25,>25)$, WHO clinical stage (I/II or III/IV), CD4 cell count $(<200$ or $\geq 200$ cells $/ \mu \mathrm{L}$ ), haemoglobin level $(\leq 10$ or $>10 \mathrm{~g} / \mathrm{dL}$ ), active $\mathrm{TB}$, and initial treatment regimen (efavirenz-based or nevirapine-based). Predictor variables which had statistical significance in the univariate analysis $(p<0.2)$ were included in the multivariate analysis. An interaction term between CD4 cell count and haemoglobin level was also added in the multivariate model. Finally, variables with $\mathrm{p}<0.05$ (statistically significant) were identified as predictors of attrition in the final model. Follow-up of patients who stopped ART medication, were LTFU or had transferred out was censored at the date of the last visit to the HF. Time to death was censored at the date of recorded death. Follow-up of retained patients was censored at the end of the study period (31 December 2015). A sensitivity analysis to estimate each transferred patient's probability of being retained in care was also performed using a multiple imputation technique. Statistical analyses were performed using SPSS software, version 21.0 (SPSS, Chicago, IL) and the SAS statistical package, version 9.2 (SAS Institute, Cary, NC).

\section{RESULTS}

\section{Participant characteristics at ART initiation}

A total of 1198 patients were included in the analysis. A majority were women $(63.4 \%)$ and their median age was 32 years. One thousand and sixty-eight $(89.1 \%)$ patients were Orthodox Christians. Approximately half (44.5\%) of the patients had no primary education. According to 
is

?

s.

s:

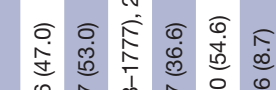

ז.

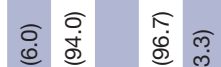

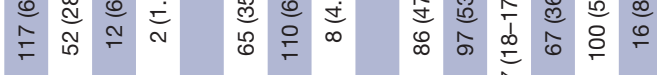

I

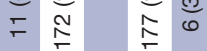

五

$\kappa$

สุ

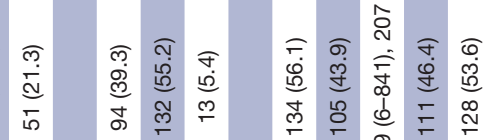

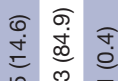

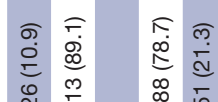

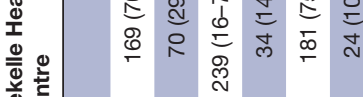

ल)

की

胥

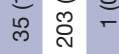

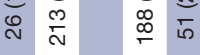

ग क

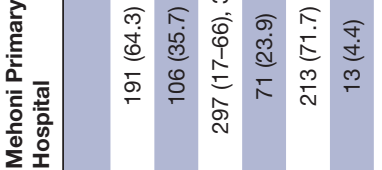

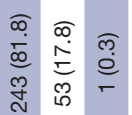

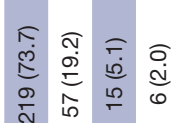

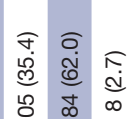

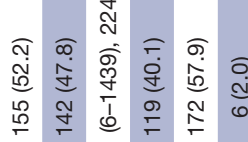

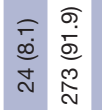

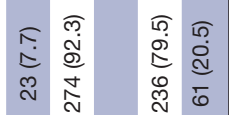

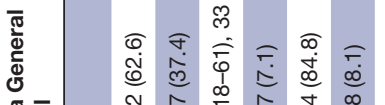

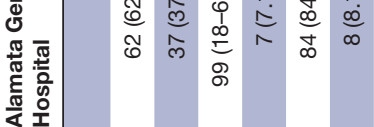

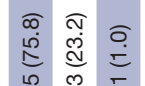

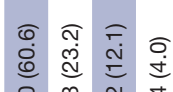

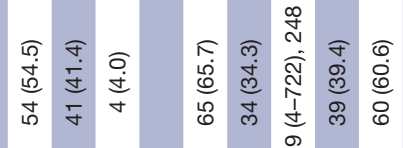

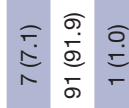

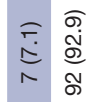

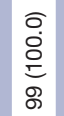

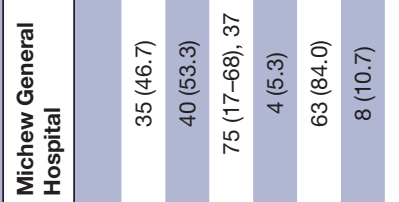

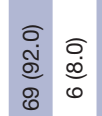

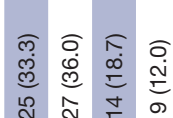

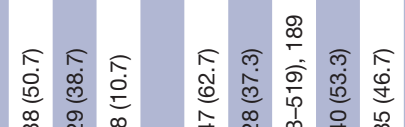

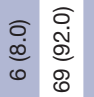

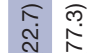

ㄷํㅇ

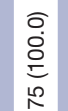

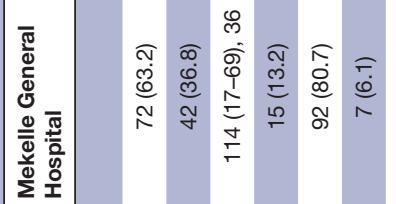

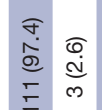

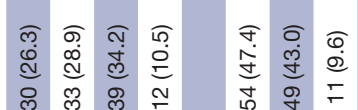

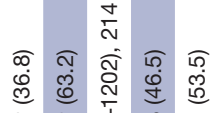

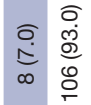

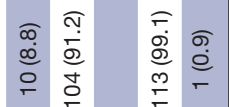

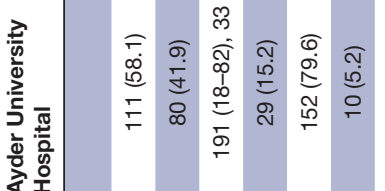

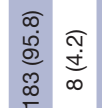

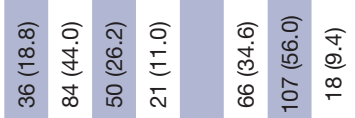

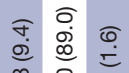

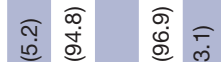

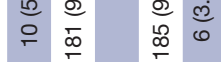

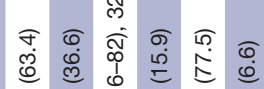

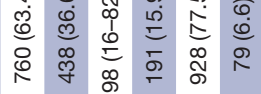

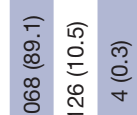

तิ

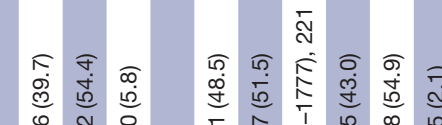

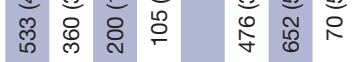

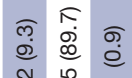

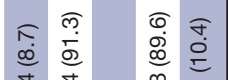
莫

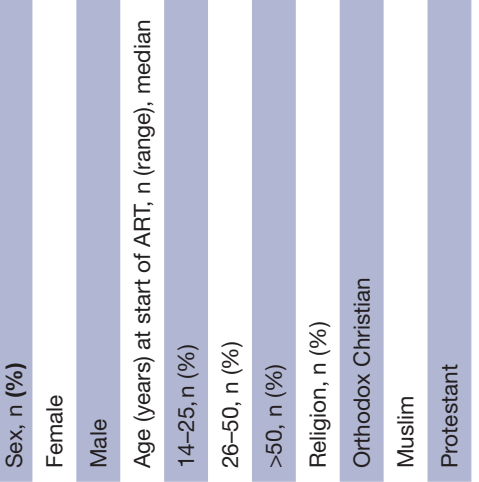

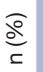

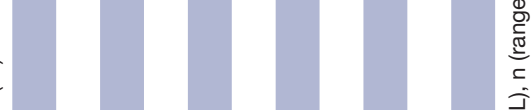

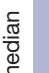

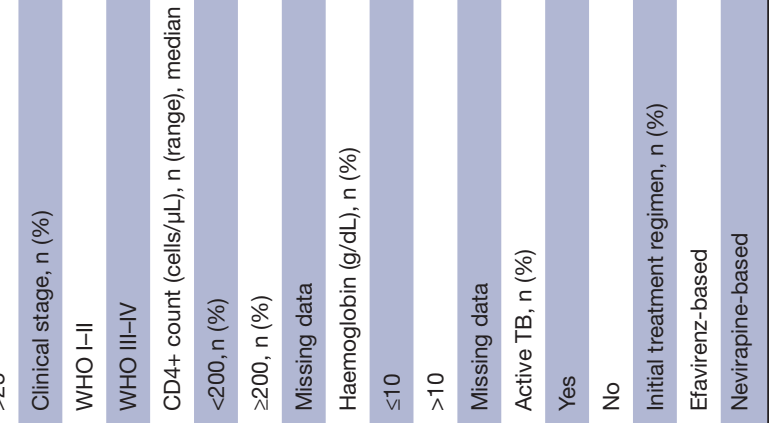




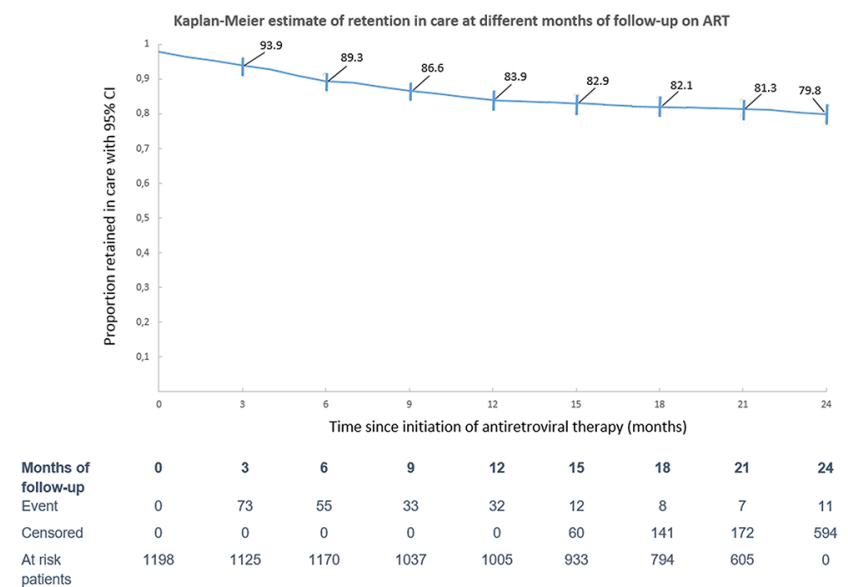

Figure 1

their BMI, $39.7 \%$ of patients were underweight. Slightly more than a half $(51.5 \%)$ were clinically symptomatic (WHO III/IV). The median CD4 count at enrolment was 221 cells $/ \mu \mathrm{L}$. At ART initiation, about $9 \%$ of patients were co-infected with active TB. Most patients $(89 \%)$ had haemoglobin values above $10 \mathrm{~g} / \mathrm{dL}$. The most common initial ART regimen was EFV-based (89.6\%) (table 1).

\section{Outcome measures after ART initiation}

Kaplan-Meier estimates of retention in care were $89.3 \%$, $83.9 \%, 82.1 \%$ and $79.8 \%$ at $6,12,18$ and 24 months of follow-up, respectively (figure 1). Attrition was mainly due to loss to follow-up, transferred-out patients and documented mortality. The loss to follow-up estimates were $3.2 \%$ at 6 months, $4.8 \%$ at 12 months, $5.8 \%$ at 18 months and $6.8 \%$ at 24 months. Transferred-out estimates were $4.6 \%$ at 6 months, $7.3 \%$ at 12 months, $8.2 \%$ at 18 months and $9.5 \%$ at 24 months. Mortality was $2.6 \%$ at 6 months, $4.0 \%$ at 12 months, $4.0 \%$ at 18 months and $4.4 \%$ at 24 months. Finally, the percentage of patients stopping ART was $0.6 \%$ after 6 months and $1.0 \%$ up to 24 months. The majority of events (deaths, loss to follow up, transferring out, ART discontinuations) occurred at 6 and 12 months of follow-up (table 2). A sensitivity analysis showed Kaplan-Meier estimates of retention of $91.7 \%$, $87.3 \%, 86.0 \%$ and $84.3 \%$ at $6,12,18$ and 24 months of follow-up, respectively (data not shown).

\section{Predictors of attrition from care}

The multivariate Cox proportional hazard model showed that male sex (HR 1.34, 95\% CI: 1.04 to 1.74), CD4 count $<200$ cells $/ \mu \mathrm{L}$ (HR 1.48, 95\% CI: 1.10 to 1.99 ) and the type of HF (Alamata Health Centre vs. Ayder Hospital: $\mathrm{HR}=2.02$, 95\% CI: 1.80 to 2.27; Alamata Hospital vs. Ayder Hospital: HR=2.81, 95\% CI: 2.48 to 3.19; Mekelle Health Centre vs. Ayder Hospital: HR=2.09, 95\% CI: 1.82 to 2.39; Mehoni Hospital vs. Ayder Hospital: $\mathrm{HR}=2.06$, 95\% CI: 1.86 to 2.28; Mekelle Hospital vs. Ayder Hospital: $\mathrm{HR}=2.15$, 95\% CI: 2.00 to 2.32; Michew Hospital vs. Ayder Hospital: HR=2.00, 95\% CI: 1.68 to 2.39 ) were significantly associated with attrition (table 3 ).

\section{DISCUSSION}

This study shows that in these HFs in Tigray, there was a progressive loss of retention in care, which had declined to only $79.8 \%$ at 24 months after ART initiation. Loss to follow-up, documented mortality and transfer out to a different HF were the main causes of attrition. In addition, the study shows that the majority of loss to follow-up and deaths occurred in the first 12 months after ART initiation. These findings are in line with previous studies from Ethiopia ${ }^{7-11}$ and other sub-Saharan African countries. $^{512}$

However, our mortality estimate was lower than estimates reported in other studies conducted in Ethiopia $^{8911}$ as well as in low- and middle-income countries. ${ }^{13}$ Melaku et al, in a multi-clinic observational study conducted in Ethiopia, showed that the cumulative incidence of death was $6 \%$ at 6 months, $7 \%$ at 12 months and $8 \%$ at 24 months. ${ }^{9}$ Another study, conducted in Ethiopia by Wubshet $e t$ al, showed that mortality was $3.7 \%, 5.6 \%$, $7.1 \%$ and $8.1 \%$ at $6,12,18$ and 24 months, respectively. ${ }^{8}$ A systematic review in low- and middle-income countries by Gupta et al found that the overall estimate of mortality at 12 months after ART initiation was $14 \%$, with the highest probability of $17 \%$ being found in sub-Saharan African countries, followed by Asia at $11 \%$, and the Americas at $7 \% .{ }^{13}$ The mortality reported in this study could be an underestimate of true mortality as we were unable to accurately ascertain patient deaths. We expect that some of the patients classified as LTFU, transferred out or stopping ART, might have died.

This study is an update of a previously published article that reported preliminary results at 1 year following ART initiation and with fewer participating HFs. ${ }^{6}$ In this study with a larger sample size, the retention in care estimate of $83.9 \%$ in the first 12 months of ART is in line with our previous findings showing a retention estimate of $85 \%$. Data show that the majority of the loss to follow-up occurred in the first year of treatment. However, comparison of the results of this study (where local CHWs attempted to trace patients who failed to return for scheduled visits) with the results of our previous study (where a structured intervention to improve retention in care was not implemented) shows a slight decrease in loss to follow-up from $5.5 \%$ to $4.8 \%$ after 1 year since ART initiation. In addition, the data on retention rate at 24 months are encouraging and suggest that the Kaplan-Meier estimate of loss to follow-up during the second year is lower (over $60 \%$ of the loss to follow-up occurred during the first year of treatment). These observations suggest that efforts to improve retention in those at high risk of loss to follow-up should be mainly focused on the initial 12 months after starting ART. A longer follow-up is needed to confirm this hypothesis.

We identified the type of HF, male sex and CD4 count $<200$ cells $/ \mu \mathrm{L}$ as important determinants of attrition from care. 


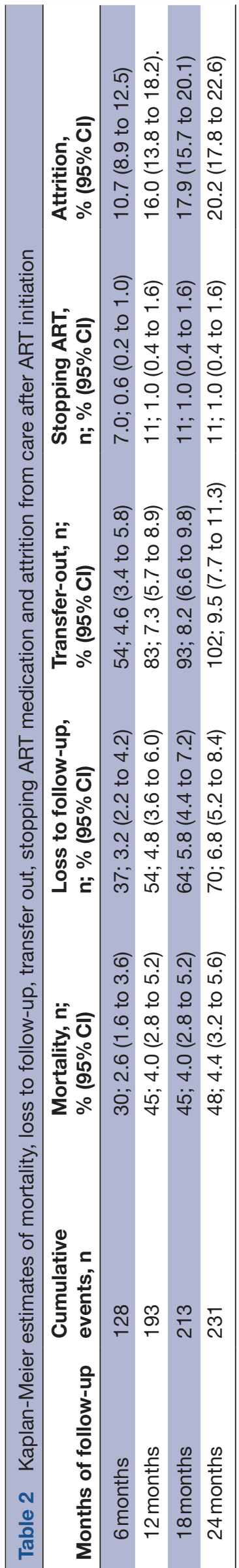

In our study, Ayder Referral Hospital showed a lower risk of attrition than the other HFs. We do not know the reason for this, but patients may have greater confidence in the health personnel at Ayder Referral Hospital as it is one of the most prestigious HFs in the country. Assefa $e t$ $a l$, in a study conducted in $55 \mathrm{HFs}$ in Ethiopia, found that retention in care varies across HFs with high, medium and low retention rates. ${ }^{10}$ In another study carried out in south Ethiopia, Teshome et al reported a higher death rate and a lower LTFU rate in health centres than in hospitals. ${ }^{14}$ However, a recent study in Ethiopia by Assefa et al found that the type of HF (health centre, secondary hospital or tertiary hospital) was associated with shortterm but not long-term retention. ${ }^{11}$ More specific studies to analyse the reasons for different retention rates among HFs are required.

Male gender is another factor independently associated with a higher rate of attrition from care. Differences in attrition from care between HIV-infected men and women have been observed in many other studies conducted in sub-Saharan Africa. ${ }^{11}{ }^{15-22}$ Some reports suggest that the reasons for lower retention among men may be due to gender differences in health-seeking behaviour, biological differences in response to ART, increased male risk for opportunistic infections and worse adherence of men to ART. Moreover, a study of eight South African ART programmes for HIV-infected adults starting ART suggested differences in mortality by gender may be due to background differences in mortality between men and women unrelated to the HIV/AIDS epidemic. ${ }^{22}$ However, women are more also likely than men to access therapy. For example, because of prevention of mother to child transmission (PMTCT) programmes, it is probable that women present at HFs for ART earlier than men. ${ }^{23} 24$

Other studies conducted in Ethiopia have also shown that a low CD4+ cell count was an independent factor associated with a higher attrition rate. ${ }^{7-9}$ This finding is also consistent with a large longitudinal ecological study conducted in nine African countries by Elus et al, which showed an association between a lower median CD4 count at ART initiation and higher 6-month attrition. ${ }^{25}$ This finding suggests that innovative HIV testing strategies should be further supported to avoid CD4 levels falling too low. According to UNAIDS, more than 14.5 of the 36.7 million PLWH at the end of 2015 did not know their HIV status. ${ }^{26}$

This study has both strengths and limitations.

One limitation is the high proportion of patients who were transferred out to another HF. Although this is common in Ethiopia, as well as in other sub-Saharan countries, as a consequence of the scaling-up of decentralisation of ART delivery, in our study transferred-out patients were not considered as retained in care because official documentation to certify that they were still on ART was not always available. Therefore, the retention rate estimate might be an underestimate of the true value.

A further limitation should be considered. Although our study is being conducted according to routine clinical 
Table 3 Cox proportional hazards model of association between baseline characteristics and attrition from care

\section{Univariate analysis, Multivariate analysis,}

$\mathrm{HR}^{\wedge}$ of attrition $(95 \% \mathrm{Cl})$

\section{$\mathrm{HR}^{\wedge}$ of attrition $(95 \% \mathrm{CI})$}

\section{Health facility}

Ayder Hospital (university hospita)

Reference

Reference

Alamata Health Centre (health centre)

$2.18(1.28 \text { to } 3.71)^{*}$

Alamata Hospital (general hospital)

2.90 (1.62 to 5.19$)$

$2.02(1.80 \text { to } 2.27)^{\star \star}$

Mekelle Health Centre (health centre)

2.08 ( 1.25 to 3.46$)$

2.81 (2.48 to 3.19$)$

Mehoni Hospital (primary hospital)

1.95 (1.18 to 3.20$)$

2.09 (1.82 to 2.39$)$

Mekelle Hospital (health centre)

2.18 (1.20 to 3.98$)$

2.06 (1.86 to 2.28$)$

Michew Hospital (general hospital)

2.27 (1.17 to 4.41$)$

2.15 (2.00 to 2.32$)$

Gender

$\begin{array}{ll}\text { Female } & \text { Reference } \\ \text { Male } & 1.51(1.16 \text { to } 1.95)^{\star} \\ \text { Age (years) } & \\ 14-25 & \text { Reference } \\ 26-50 & 0.94(0.66 \text { to } 1.33) \\ >50 & 1.11(0.63 \text { to } 1.96)\end{array}$

Educational status

\begin{tabular}{|c|c|c|}
\hline No education & Reference & - \\
\hline Primary & 1.02 (0.75 to 1.38$)$ & \\
\hline Secondary & 1.11 (0.77 to 1.59$)$ & \\
\hline Tertiary & 0.99 (0.61 to 1.60$)$ & \\
\hline \multicolumn{3}{|l|}{ Religion } \\
\hline Orthodox Christian & Reference & - \\
\hline Other religions & 1.05 (0.69 to 1.59$)$ & \\
\hline \multicolumn{3}{|l|}{$\mathrm{BMI}\left(\mathrm{kg} / \mathrm{m}^{2}\right)$} \\
\hline $18.6-25$ & Reference & Reference \\
\hline$\leq 18.5$ & $1.67(1.28 \text { to } 2.17)^{\star}$ & 1.40 (0.99 to 1.98$)$ \\
\hline$>25$ & 0.69 (0.34 to 1.42$)$ & 0.79 (0.32 to 1.98$)$ \\
\hline \multicolumn{3}{|l|}{ Clinical stage } \\
\hline WHO I-II & Reference & Reference \\
\hline WHO III-IV & $1.30(1.01 \text { to } 1.69)^{*}$ & 1.10 (0.77 to 1.58$)$ \\
\hline \multicolumn{3}{|l|}{ Active TB } \\
\hline No & Reference & Reference \\
\hline Yes & $1.93(1.33 \text { to } 2.80)^{\star}$ & 1.39 (0.92 to 2.10$)$ \\
\hline \multicolumn{3}{|c|}{ Initial treatment regimen } \\
\hline Efavirenz-based & Reference & - \\
\hline Nevirapine-based & 1.17 (0.80 to 1.73$)$ & \\
\hline \multicolumn{3}{|l|}{ CD4 count (cells $/ \mu \mathrm{L})$} \\
\hline$\geq 200$ & Reference & Reference \\
\hline$<200$ & $1.68(1.29 \text { to } 2.19)^{\star}$ & $1.48(1.10 \text { to } 1.99)^{* \star} ; \S$ \\
\hline \multicolumn{3}{|l|}{ Haemoglobin (g/dL) } \\
\hline$>10$ & Reference & Reference \\
\hline$\leq 10$ & $1.40(0.98 \text { to } 2.13)^{\star}$ & 1.40 (0.96 to 2.04$) \S$ \\
\hline
\end{tabular}

${ }^{* *} p$ Value $<0.05$

$\wedge$ Hazard ratios estimated using robust sandwich estimators for variance to account for within-HF correlation.

${ }^{*} \mathrm{p}$ Value $<0.2$.

$\S \mathrm{HR}$ adjusted for interaction term between CD4 count and haemoglobin value 
practice, the fact that patients were aware that they are part of a research study may have improved their level of retention.

Moreover, although CHWs are regularly involved in the participating HFs, additional CHW-based interventions for patient tracing and counselling, as implemented in the CASA project, can lead to increased retention as compared with standard practice. However, we were not able to estimate the impact on retention of the activities of the CHWs since there was no control group.

In addition, despite the 2-year follow-up, the observation period is still too short to properly evaluate the long-term retention rate, but the study is ongoing and is planned to last at least 5 years.

The strengths of this study are its multi-site and prospective design, the quality of data, and the intensive training of the study staff.

Based on our results, we believe that combination strategies to address the many factors affecting patient retention need to be implemented, while more research on innovative models of care is needed. The different levels of attrition from care observed in our study among different types and locations of HFs suggest that action to decrease inequalities in the quality of care among HFs should be a priority for policy makers. Observed attrition differences between genders suggest that new specific interventions designed for women and men should also be explored separately. Interventions aimed at networking clinical centres and developing systems for tracking patients who transfer out should be implemented. Finally, the active involvement of CHWs in specific activities may have improved retention in care in our study. Therefore, we believe that the active involvement of well-trained CHWs should be further encouraged. $^{27-38}$

Acknowledgements We thank all the patients participating in this study, the health facilities, the community-based organisations and the CASA project team.

Contributors All authors contributed to this paper.

Funding This work was supported by the Italian Ministry of Health-Department of Prevention and Innovation, Rome, Italy; the Italian Development Cooperation; EDCTP2 Participating States' Initiated Activity (PSIA-2017-1208).

Competing interests None declared.

Patient consent Obtained.

Ethics approval Ethics approval was obtained from Mekelle University College of Health Sciences Research and Community Service Council. All patients provided written informed consent. For patients aged 14 to 18 the informed consent was signed by adult relatives acting as guardians (immediate families, e.g. father or mother or next of kin) and not by the patients themselves.

Provenance and peer review Not commissioned; externally peer reviewed.

Open Access This is an Open Access article distributed in accordance with the Creative Commons Attribution Non Commercial (CC BY-NC 4.0) license, which permits others to distribute, remix, adapt, build upon this work non-commercially, and license their derivative works on different terms, provided the original work is properly cited and the use is non-commercial. See: http://creativecommons.org/ licenses/by-nc/4.0/

(c) Article author(s) (or their employer(s) unless otherwise stated in the text of the article) 2017. All rights reserved. No commercial use is permitted unless otherwise expressly granted.

\section{REFERENCES}

1. UNAIDS. Fact sheet 2016: Global Statistics 2015. 2015 http://www. unaids.org/sites/default/files/media_asset/20150901_FactSheet_ 2015_en.pdf

2. UNAIDS. Global AIDS update. 2016 http://www.unaids.org/sites/ default/files/media_asset/global-AIDS-update-2016_en.pdf

3. World Health Organization (WHO). Global Health Observatory data repository, http://apps.who.int/gho/data/?theme=main

4. World Health Organization (WHO). Consolidated guidelines on the use of antiretroviral drugs for treating and preventing HIV infection. 2016 http://apps.who.int/iris/bitstream/10665/208825/1/ 9789241549684_eng.pdf?ua $=1$

5. Fox MP, Rosen S. Patient retention in antiretroviral therapy programs up to three years on treatment in sub-Saharan Africa, 2007-2009: systematic review. Trop Med Int Health 2010;15(Suppl 1):1-15.

6. Bucciardini R, Fragola V, Abegaz T, et al. Retention in care of adult HIV patients initiating antiretroviral therapy in Tigray, Ethiopia: a prospective observational cohort study. PLoS One 2015;10:e0136117.

7. Mekuria LA, Prins JM, Yalew AW, et al. Retention in HIV care and predictors of attrition from care among HIV-infected adults receiving combination anti-retroviral therapy in Addis Ababa. PLoS One 2015;10:e0130649.

8. Wubshet M, Berhane $Y$, Worku A, et al. High loss to follow up and early mortality create substantial reduction in patient retention at antiretroviral treatment program in North-West Ethiopia. International Scholarly Research Network (ISRN) AIDS 2012;2012:721720.

9. Melaku Z, Lamb MR, Wang C, et al. Characteristics and outcomes of adult Ethiopian patients enrolled in HIV care and treatment: a multiclinic observational study. BMC Public Health 2015;15:462.

10. Assefa $Y$, Kiflie A, Tesfaye D, et al. Outcomes of antiretroviral treatment program in Ethiopia: retention of patients in care is a major challenge and varies across health facilities. BMC Health Serv Res 2011;11:81.

11. Assefa $Y$, Lynen L, Kloos $\mathrm{H}$, et al. Brief report: long-term outcomes and their determinants in patients on antiretroviral treatment in Ethiopia, 2005/6-2011/12: a retrospective cohort study. J Acquir Immune Defic Syndr 2015;70:414-9.

12. Rasschaert $F$, Koole $O$, Zachariah $R$, et al. Short and long term retention in antiretroviral care in health facilities in rural Malawi and Zimbabwe. BMC Health Serv Res 2012;12:444.

13. Gupta A, Nadkarni G, Yang WT, et al. Early mortality in adults initiating antiretroviral therapy (ART) in low- and middle-income countries (LMIC): a systematic review and meta-analysis. PLoS One 2011;6:e28691

14. Teshome W, Belayneh M, Moges M, et al. Do loss to follow-up and death rates from ART care vary across primary health care facilities and hospitals in south Ethiopia? A retrospective follow-up study. HIV AIDS 2015;7:167-74.

15. Centers for Disease Control and Prevention (CDC). Differences between HIV-Infected men and women in antiretroviral therapy outcomes - six African countries, 2004-2012. MMWR Morb Mortal Wkly Rep 2013;62:945-52 http://www.cdc.gov/mmwr/preview/ $\mathrm{mmwrhtml} / \mathrm{mm} 6247 \mathrm{a} 2 . \mathrm{htm}$.

16. Muula AS, Ngulube TJ, Siziya S, et al. Gender distribution of adult patients on highly active antiretroviral therapy (HAART) in Southern Africa: a systematic review. BMC Public Health 2007;7:63.

17. Taylor-Smith K, Tweya H, Harries A, et al. Gender differences in retention and survival on antiretroviral therapy of HIV-1 infected adults in Malawi. Malawi Med $J$ 2010;22:49-56.

18. Cornell M, Myer L, Kaplan R, et al. The impact of gender and income on survival and retention in a South African antiretroviral therapy programme. Trop Med Int Health 2009;14:722-31.

19. Theuring S, Mugenyi K, Rubaihayo J, et al. Antiretroviral therapy programme retention and outcomes after 12 months in a retrospective patient cohort in Fort Portal, Uganda: the ongoing challenge of male ART performance. J AIDS Clin Res 2015;6:423.

20. Takarinda KC, Harries AD, Shiraishi RW, et al. Gender-related differences in outcomes and attrition on antiretroviral treatment among an HIV-infected patient cohort in Zimbabwe: 2007-2010. Int J Infect Dis 2015;30:98-105.

21. Maskew M, Brennan AT, Westreich D, et al. Gender differences in mortality and CD4 count response among virally suppressed HIVpositive patients. J Womens Health 2013;22:113-20.

22. Cornell M, Schomaker M, Garone DB, et al. Gender differences in survival among adult patients starting antiretroviral therapy in South Africa: a multicentre cohort study. PLoS Med 2012;9:e1001304.

23. Braitstein P, Boulle A, Nash D, et al. Gender and the use of antiretroviral treatment in resource-constrained settings: findings from a multicenter collaboration. J Womens Health 2008;17:47-55. 
24. May M, Boulle A, Phiri S, et al. Prognosis of patients with HIV1 infection starting antiretroviral therapy in sub-Saharan Africa: a collaborative analysis of scale-up programmes. Lancet 2010;376:449-57.

25. Elul B, Saito S, Chung H, et al. Attrition from HIV treatment programs in Africa: a longitudinal ecological analysis using data from 307144 patients initiating antiretroviral therapy between 2005 and 2010. Clin Infect Dis 2017;64:1309-16.

26. UNAIDS: Prevention gap report. 2016 http://www.unaids.org/sites/ default/files/media_asset/2016-prevention-gap-report_en.pdf

27. Grimsrud A, Sharp J, Kalombo C, et al. Implementation of community-based adherence clubs for stable antiretroviral therapy patients in Cape Town, South Africa. J Int AIDS Soc 2015;18:19984.

28. Nglazi MD, Lawn SD, Kaplan R, et al. Changes in programmatic outcomes during 7 years of scale-up at a community-based antiretroviral treatment service in South Africa. J Acquir Immune Defic Syndr 2011;56:e1-e8.

29. Jaffar S, Amuron B, Foster S, et al. Rates of virological failure in patients treated in a home-based versus a facility-based HIV-care model in Jinja, southeast Uganda: a cluster-randomised equivalence trial. Lancet 2009;374:2080-9.

30. Kipp W, Konde-Lule J, Rubaale T, et al. Comparing antiretroviral treatment outcomes between a prospective community-based and hospital-based cohort of HIV patients in rural Uganda. BMC Int Health Hum Rights 2011;11(Suppl 2):S12.

31. Mwai GW, Mburu G, Torpey K, et al. Role and outcomes of community health workers in HIV care in sub-Saharan Africa: a systematic review. J Int AIDS Soc 2013;16:18586.
32. Selke HM, Kimaiyo S, Sidle JE, et al. Task-shifting of antiretroviral delivery from health care workers to persons living with HIV/AIDS: clinical outcomes of a community-based program in Kenya. $J$ Acquir Immune Defic Syndr 2010;55:483-90.

33. Kredo T, Ford N, Adeniyi FB, et al. Decentralising HIV treatment in lower- and middle-income cuntries. Cochrane Database Syst Rev 2013;6:CD009987.

34. Bemelmans M, Baert S, Goemaere E, et al. Community-supported models of care for people on HIV treatment in sub-Saharan Africa. Trop Med Int Health 2014;19:968-77.

35. Grimsrud A, Lesosky M, Kalombo C, et al. Implementation and operational research: community-based adherence clubs for the management of stable antiretroviral therapy patients in Cape Town, South Africa: a cohort study. J Acquir Immune Defic Syndr 2016;71:e16-23.

36. Decroo T, Rasschaert F, Telfer B, et al. Community-based antiretroviral therapy programs can overcome barriers to retention of patients and decongest health services in sub-Saharan Africa: a systematic review. Int Health 2013;5:169-79.

37. Fulton BD, Scheffler RM, Sparkes SP, et al. Health workforce skill mix and task shifting in low income countries: a review of recent evidence. Hum Resour Health 2011;9:1.

38. Van Damme W, Kober K, Kegels G. Scaling-up antiretroviral treatment in Southern African countries with human resource shortage: how will health systems adapt? Soc Sci Med 2008;66:2108-21. 